\title{
Clinicopathological characteristics and prognosis of breast cancer patients with type 2 diabetes mellitus
}

\author{
DE HE ${ }^{1}$, JING-WEN BAI ${ }^{1,2}$, JING LIU $^{2}$, CAI-WEN DU ${ }^{3}$, WEN-HE HUANG $^{1}$ and GUO-JUN ZHANG ${ }^{1,2}$ \\ ${ }^{1}$ The Breast Center, Cancer Hospital of Shantou University Medical College; \\ ${ }^{2}$ Cancer Research Center of Shantou University Medical College; ${ }^{3}$ Department of Breast Medical Oncology, \\ Cancer Hospital of Shantou University Medical College, Shantou, Guangdong 515041, P.R. China
}

Received October 17, 2014; Accepted December 23, 2014

DOI: $10.3892 /$ mco.2015.522

\begin{abstract}
Type 2 diabetes mellitus (T2DM) can increase the risk of several common cancers, including breast cancer (BC). The purpose of the present study was to investigate the clinicopathological features and prognosis of $\mathrm{BC}$ patients with or without T2DM. Seventy-eight patients were diagnosed with T2DM prior to the diagnosis of BC in the Cancer Hospital of Shantou University Medical College (Shantou, China) between 2002 and 2008. A total of 300 BC patients without T2DM were randomly selected as study controls during the same period. The clinicopathological characteristics, overall survival (OS) and disease-free survival (DFS) rates of these two groups were compared. Fifty-five BC patients and 133 control patients with T2DM were $>50$ years old (70.5 and $44.3 \%$, respectively). There were more T2DM BC patients with body mass index $(\mathrm{BMI}) \geq 25 \mathrm{~kg} / \mathrm{m}^{2}$ (46.2 vs. $23.3 \%)$ and these patients had a higher rate of lymph node involvement (67.9 vs. $55.0 \%$ ). The DFS of the two groups was 32.1 vs. $22.3 \%$. The OS of the two groups was 24.4 vs. $13.7 \%$. Following adjustment for BMI, tumor-node metastasis stage and stratification of age, the relapse risk of T2DM BC patients was $>2$-fold higher than that of the control group in the estrogen receptor/progesterone receptor (ER/PR)-positive patients. In Her-2-negative BC patients, the relapse risk of T2DM patients was 2.237-fold higher than that of the non-T2DM patients. In conclusion, T2DM BC patients were significantly older and more likely to be overweight, and had more lymph nodes involvement. T2DM was associated
\end{abstract}

Correspondence to: Professor Guo-Jun Zhang, Cancer Research Center of Shantou University Medical College, 7 Raoping Road, Shantou, Guangdong 515041, P.R. China

E-mail: guoj_zhang@yahoo.com

Mr Wen-He Huang, The Breast Center, Cancer Hospital of Shantou University Medical College, 22 Xinling Road, Shantou, Guangdong 515041, P.R. China

E-mail: huangwenhe2009@163.com

Key words: breast cancer, clinicopathological characteristics, type 2 diabetes mellitus, overall survival rate, disease-free survival rate with poor prognosis in ER/PR positive or Her-2-negative BC patients.

\section{Introduction}

Diabetes mellitus is a metabolic disease and a major health issue worldwide. The population of diabetes patients increased from 153 million in 1980, to 347 million in 2008 (1). Type 2 diabetes mellitus (T2DM), which accounts for $>90 \%$ of all diagnosed cases of diabetes mellitus, is characterized by insulin resistance and hyperinsulinemia. The association between diabetes mellitus and cancer has been widely reported (2). Diabetes mellitus is now recognized as a risk factor for different types of cancers, including liver, pancreas, endometrium, colon/rectum, breast and bladder cancer, although it is associated with a reduced risk of prostate cancer (3).

Breast cancer $(\mathrm{BC})$ is the most common malignant tumor in females worldwide, accounting for $23 \%$ (1.38 million) of all new cancer cases and $14 \%(458,400)$ of the total cancer fatalities in 2008 (4). Meta-analysis have showed that females with a history of diabetes had a $20 \%$ increased risk of BC compared to females without diabetes (5). Michels et al (6) found that females with type 2 diabetes had a modestly elevated incidence of $\mathrm{BC}$ compared to females without diabetes and this association was more predominant among estrogen receptor (ER)-positive BC patients.

Numerous epidemiological studies have demonstrated that T2DM increased BC risk, but whether T2DM adversely affects the prognosis of $\mathrm{BC}$ remains unclear. The direct biological effects of diabetes on $\mathrm{BC}$ patients are difficult to define, mainly due to the presence of confounding factors, such as obesity, old age, comorbidity and differences in screening method or treatment. In the present study, T2DM $\mathrm{BC}$ patients were older, more likely to be post-menopausal, overweight or obese, had a higher incidence of involved lymph nodes and were at a more advanced tumor stage. At least two studies had reported similar clinicopathological features and found that BC patients with T2DM tend to be older, at a much more advanced tumor-node metastasis (TNM) stage and had more lymph node involvement $(7,8)$. The differences between the T2DM and non-T2DM groups may be partially attributed to the differences in social and economic status: 
BC patients with T2DM are generally poorly educated, older, have complications from other diseases and usually have fewer opportunities for physical examination. Doctors often focus on the treatment of diabetes and its complication, and ignore the diagnosis of BC. Thus, the patients with T2DM are usually diagnosed at a more advanced tumor stage (9). T2DM and BC share some common risk factors, such as old age, obesity, high-fat diet and less physical activity, and these factors may influence prognosis. Peairs et al (10) found BC patients with diabetes had a significantly higher all-cause mortality risk (1.49-fold). However, the extent of the impact of $\mathrm{T} 2 \mathrm{DM}$ on the prognosis of $\mathrm{BC}$ patients remains poorly understood.

The present study aimed to identify the clinicopathological features of BC patients with T2DM and to determine the prognostic factors for these patients.

\section{Patients and methods}

Patient characteristics. A total of $378 \mathrm{BC}$ patients were enrolled in the retrospective study. All the patients were diagnosed as BC with stage I-III and received surgery at the Cancer Hospital of Shantou University Medical College (Shantou, China) between 2002 and 2008. Among these patients, prior to diagnosis of BC, 78 were diagnosed with T2DM, while the remaining $300 \mathrm{BC}$ patients without T2DM were randomly selected as the control group. Of all the patients, 325 received modified-radical mastectomy, 28 received radical mastectomy and 25 underwent breast-conserving surgery. Patients were 23-88 years old with a median age of 50 years old. All the patients were divided into two groups: T2DM BC group and non-T2DM BC group. Written informed consent was obtained for each patient and the study was approved by the Hospital Research Ethics Committee.

The overall survival (OS) rate was calculated from the date of surgery to the date of mortality or last follow-up visit. The disease-free survival (DFS) rate was calculated from the date of surgery to the date of relapse. By the end of January 2011, the median follow-up time was 42 months (range, 5-120 months) and 60 patients (15.9\%) had succumbed during the follow-up period, of which $31.7 \%$ (19/60) and $68.3 \%$ (41/60) of the mortalities were T2DM BC and non-T2DM BC patients, respectively. Ninety-two patients $(24.3 \%)$ had recurrence during the follow-up time, of which $27.2 \%$ (25/92) were T2DM BC patients and 72.8\% (67/92) were non-T2DM BC patients.

Immunohistochemistry. Serial formalin-fixed and paraffin-embedded tissues were sectioned at $4-\mu \mathrm{m}$ thickness, deparaffinized and rehydrated in a gradient of high percentage ethanol to distilled water. For quenching endogenous peroxidase activity, sections were immersed in $3 \%$ hydrogen peroxide for $15 \mathrm{~min}$ at room temperature. Antigen retrieval involved boiling in $10 \mathrm{mmol} / \mathrm{l}$ sodium citrate buffer ( $\mathrm{pH}$ 6) for $3 \mathrm{~min}$ in a microwave, followed by cooling to room temperature. Sections were subsequently incubated with the primary antibody at $4^{\circ} \mathrm{C}$ overnight, washed three times in phosphate-buffered saline for $5 \mathrm{~min}$ and incubated with horseradish peroxidase (HRP)-conjugated goat anti-mouse/rabbit immunoglobulin G (ZSGB-Bio, Beijing,
China) at room temperature for $30 \mathrm{~min}$. Bound antibodies were detected by sequential incubation with anti-rabbit Envision ${ }^{\mathrm{TM}}$ HRP-conjugated polymer (Dako Finland Oy, Helsinki, Finland), followed by 3,3'-diaminobenzidine tetra-hydrochloride staining. Sections were lightly counterstained with hematoxylin.

The antibodies used were as follows: ER $\alpha$ (sc-543), progesterone receptor (PR; sc53423) (both from Santa Cruz Biotechnology, Inc., Santa Cruz, CA, USA) and Her-2 (RB-9040-P; LabVision Corp., Fremont, CA, USA).

Statistical analysis. SPSS version 17.0 software (SPSS, Inc., Chicago, IL, USA) was used for statistical analysis. Clinicopathological characteristics of patients were compared using the $\chi^{2}$ test or Fisher's exact test. The Kaplan-Meier method and log-rank test were used for survival rate analysis. In order to avoid confounding factors, Cox proportional hazard models were used to obtain hazard ratios (HR) for the T2DM and non-T2DM groups following adjusting for TNM stage, body mass index (BMI) and age stratification in the subgroup analysis.

\section{Results}

Clinicopathological characteristics between the T2DM $B C$ and non-T2DM BC groups. The general information of the patients is shown in Table I. Patients in the T2DM $\mathrm{BC}$ group were older $(\mathrm{P}<0.001)$ and the postmenopausal proportion was higher (67.9 vs. $42.0 \%, \mathrm{P}<0.001)$. The World Health Organization defines normal weight as having a BMI ranging, $18.5-24.9 \mathrm{~kg} / \mathrm{m}^{2}$; overweight is defined as having a BMI $>25 \mathrm{~kg} / \mathrm{m}^{2}$. Therefore, patients were divided into two groups according to BMI, using $25 \mathrm{~kg} / \mathrm{m}^{2}$ as a cut-off point in the study. Patients with T2DM were likely to be overweight or obese (BMI, $\geq 25 \mathrm{~kg} / \mathrm{m}^{2} ; 46.2$ vs. $\left.23.3 \%, \mathrm{P}<0.001\right)$. In the T2DM BC group, the proportion of patients with TNM stage III was higher than that of the non-T2DM BC group (60.3 vs. $40.0 \%, \mathrm{P}=0.001)$. Lymph nodes involvement was significantly higher in T2DM BC patients (67.9 vs. $55.0 \%$, $\mathrm{P}=0.001)$. T2DM patients had a higher proportion of triple-negative $\mathrm{BC}$ than that of the non-T2DM patients (21.8 vs. $13.3 \%, \mathrm{P}=0.02$ ). There was no statistical difference in tumor size, ER, PR and Her-2 status between the two groups.

Survival rate analysis between the T2DM BC and non-T2DM $B C$ groups. The probability of 3-year DFS for the T2DM BC and non-T2DM BC groups was 81.1 and $92.9 \%$ and that of 5-year DFS was 67.8 and $90.4 \%$, respectively. The DFS for the T2DM group was significantly worse than that of the non-T2DM group (Fig. 1A, $\mathrm{P}=0.028$ ). The probability of 3-year OS for the T2DM BC and non-T2DM BC groups was 84.5 and 93.9\% and for 5-year OS was 70.3 and $93.2 \%$, respectively. Similarly, the OS of the T2DM BC group was worse than that of the non-DM BC group (Fig. $1 \mathrm{~B}, \mathrm{P}=0.012$ ).

Multivariate analysis of DFS and OS in subgroups of patients with invasive breast carcinoma. Following adjustment for BMI, TNM stage and stratification by age, patients with T2DM tended to have a 1.483 -fold increase in the 
Table I. Comparison of clinicopathological parameters in breast cancer patient groups with or without type 2 diabetes mellitus (T2DM).

\begin{tabular}{|c|c|c|c|c|}
\hline $\begin{array}{l}\text { Clinicopathological } \\
\text { features }\end{array}$ & $\begin{array}{c}\text { T2DM } \\
\text { group, n }(\%)\end{array}$ & $\begin{array}{l}\text { Non-T2DM } \\
\text { group, n }(\%)\end{array}$ & $\chi^{2}$ & P-value \\
\hline \multicolumn{5}{|l|}{ Age, years } \\
\hline$<50$ & $23(12.1)$ & 167 (87.9) & 16.97 & 0.001 \\
\hline$\geq 50$ & $55(29.3)$ & 133 (70.7) & & \\
\hline \multicolumn{5}{|l|}{ Menopausal status } \\
\hline Pre- & $25(12.6)$ & $174(87.4)$ & 16.72 & $<0.001$ \\
\hline Post- & $53(29.6)$ & $126(70.4)$ & & \\
\hline \multicolumn{5}{|l|}{$\mathrm{BMI}, \mathrm{kg} / \mathrm{m}^{2}$} \\
\hline$<25$ & $42(15.4)$ & $230(84.6)$ & 15.98 & $<0.001$ \\
\hline$\geq 25$ & $36(36.0)$ & $64(64.0)$ & & \\
\hline \multicolumn{5}{|l|}{ Tumor size } \\
\hline $\mathrm{T}_{1-2}$ & $45(17.8)$ & 208 (82.2) & 3.790 & 0.052 \\
\hline $\mathrm{T}_{3-4}$ & $33(26.4)$ & $92(73.6)$ & & \\
\hline \multicolumn{5}{|l|}{$\begin{array}{l}\text { Lymph nodes } \\
\text { involved }\end{array}$} \\
\hline Negative & $25(15.6)$ & $135(84.4)$ & 4.252 & 0.039 \\
\hline Positive & $53(24.3)$ & 165 (75.7) & & \\
\hline \multicolumn{5}{|l|}{ AJCC stage } \\
\hline I-II & $31(14.7)$ & $180(85.3)$ & 10.30 & 0.001 \\
\hline III & $47(28.1)$ & $120(71.9)$ & & \\
\hline \multicolumn{5}{|l|}{ ER } \\
\hline Negative & $29(23.2)$ & $96(76.8)$ & 0.750 & 0.386 \\
\hline Positive & $49(19.4)$ & 204 (80.6) & & \\
\hline \multicolumn{5}{|l|}{ PR } \\
\hline Negative & $35(21.2)$ & $130(78.8)$ & 0.060 & 0.807 \\
\hline Postitive & $43(20.2)$ & $170(79.8)$ & & \\
\hline \multicolumn{5}{|l|}{ Her-2 } \\
\hline Negative & $49(20.7)$ & $188(79.3)$ & 0.01 & 0.980 \\
\hline Positive & $29(20.6)$ & $112(79.4)$ & & \\
\hline \multicolumn{5}{|l|}{ Histological grade } \\
\hline I & $21(17.4)$ & $100(82.6)$ & 1.169 & 0.280 \\
\hline II-III & $57(22.2)$ & $200(77.8)$ & & \\
\hline \multicolumn{5}{|l|}{ Molecular subtype } \\
\hline Luminal A & $32(17.6)$ & $150(82.4)$ & 4.531 & 0.210 \\
\hline Luminal B & $17(23.3)$ & $56(76.7)$ & & \\
\hline $\begin{array}{l}\text { Her- } 2 \\
\text { overexpression }\end{array}$ & $12(18.2)$ & $54(81.8)$ & & \\
\hline TNBC & $17(29.8)$ & $40(70.2)$ & & \\
\hline
\end{tabular}

BMI, body mass index; AJCC, American Joint Committee on Cancer; ER, estrogen receptor; PR, progesterone receptor; Her-2, human epidermal growth factor 2; TNBC, triple-negative breast cancer.

relative risk of cancer recurrence compared to patients without T2DM [95\% confidence interval (CI), 0.909-2.420], although without statistical significance $(\mathrm{P}=0.114)$. For the ER/PR-positive group, the recurrence risk of $\mathrm{BC}$ with T2DM was $>2$-fold higher than that of the ER/PR-positive
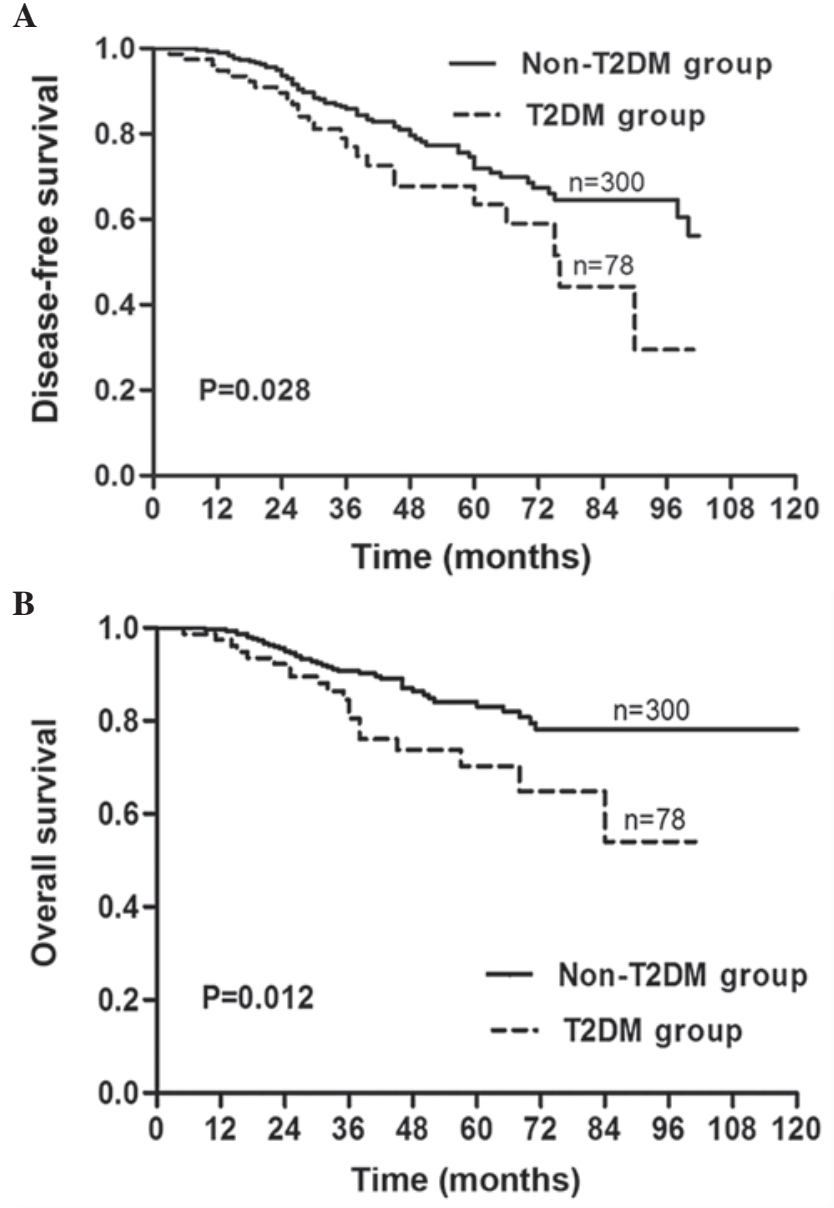

Figure 1. Type 2 diabetes mellitus (T2DM) was associated with poor disease-free (DFS) and overall survival (OS) rates in patients with invasive breast carcinoma. (A) DFS and (B) OS were calculated by Kaplan-Meier survival analysis between the patient group with (dotted line) or without T2DM (solid line).

non-T2DM BC patients (HR=2.062; 95\% CI, 1.118-3.804; $\mathrm{P}=0.021)$. In the Her-2-negative group, the relapse risk of $\mathrm{BC}$ with T2DM was 2.237-fold higher compared to the non-T2DM BC patients $(\mathrm{HR}=2.237$; 95\% CI, 1.186-4.220; $\mathrm{P}=0.013)$. In the comparison of the other parameters, there was no statistical significance between T2DM and non-T2DM patients (Table II).

Following adjustment for BMI, TNM stage and stratification of age in the subgroups, Cox regression analyses showed that ER/PR-positive patients with T2DM tended to have a 1.688-fold increase in tumor recurrence compared to the patients without T2DM (95\% CI, 0.940-3.032), but the difference was not statistically significant $(\mathrm{P}=0.079)$. ER/PR-positive patients with T2DM showed a 2.255-fold higher mortality risk compared to those without T2DM. However, Cox analysis showed that in either ER/PR-negative patients or Her-2-positive patients, the presence of T2DM was not significantly associated with a poor prognosis (Table III).

\section{Discussion}

The Han Chinese population in Guangdong, South China, can be divided into three public populations; Cantonese, Hakka and Chaoshannese. Chaoshannese has its own customs and 
Table II. Cox proportional regression analysis on the disease-free survival rate of patients with invasive breast carcinoma stratified by the diabetes status in the subgroup.

\begin{tabular}{|c|c|c|c|c|c|}
\hline Variables & T2DM group & Non-T2DM group & HR & $95 \% \mathrm{CI}$ & P-value \\
\hline \multicolumn{6}{|c|}{ Menopause status } \\
\hline Pre- & $8 / 25$ & $38 / 174$ & 2.082 & $0.932-4.652$ & 0.074 \\
\hline Post- & $17 / 53$ & $29 / 126$ & 1.252 & $0.668-2.346$ & 0.483 \\
\hline \multicolumn{6}{|l|}{$\mathrm{BMI}, \mathrm{kg} / \mathrm{m}^{2}$} \\
\hline$<25$ & $13 / 42$ & $54 / 230$ & 1.400 & $0.751-2.611$ & 0.289 \\
\hline$\geq 25$ & $12 / 36$ & $13 / 70$ & 1.805 & $0.776-4.200$ & 0.171 \\
\hline \multicolumn{6}{|l|}{ pT } \\
\hline $\mathrm{T}_{1-2}$ & $13 / 45$ & $43 / 208$ & 1.332 & $0.699-2.538$ & 0.384 \\
\hline $\mathrm{T}_{3-4}$ & $12 / 33$ & $24 / 92$ & 1.872 & $0.857-4.091$ & 0.116 \\
\hline \multicolumn{6}{|c|}{ Lymph nodes } \\
\hline Negative & $5 / 25$ & 24/135 & 1.039 & $0.375-2.876$ & 0.942 \\
\hline Positive & $20 / 53$ & $43 / 165$ & 1.686 & $0.961-2.959$ & 0.069 \\
\hline \multicolumn{6}{|l|}{ AJCC stage } \\
\hline I-II & $8 / 31$ & $33 / 180$ & 1.383 & $0.622-3.075$ & 0.426 \\
\hline III & $17 / 47$ & $34 / 120$ & 1.588 & $0.849-2.972$ & 0.148 \\
\hline \multicolumn{6}{|l|}{ Grade } \\
\hline 1 & $5 / 21$ & $21 / 100$ & 1.510 & $0.504-4.530$ & 0.462 \\
\hline $2-3$ & $20 / 57$ & $46 / 200$ & 1.352 & $0.778-2.349$ & 0.285 \\
\hline \multicolumn{6}{|c|}{ ER/PR status } \\
\hline Negative & $10 / 29$ & $27 / 94$ & 0.730 & $0.317-1.684$ & 0.461 \\
\hline Positive & $15 / 49$ & $40 / 206$ & 2.062 & $1.118-3.804$ & 0.021 \\
\hline \multicolumn{6}{|l|}{ Her-2 status } \\
\hline Negative & $16 / 49$ & $37 / 188$ & 2.237 & $1.186-4.220$ & 0.013 \\
\hline Positive & $9 / 29$ & $30 / 112$ & 0.685 & $0.375-1.904$ & 0.685 \\
\hline Overall & $25 / 78$ & $67 / 300$ & 1.483 & $0.909-2.420$ & 0.114 \\
\hline
\end{tabular}

T2DM, type 2 diabetes mellitus; HR, hazard ratio; CI, confidence interval; BMI, body mass index; AJCC, American Joint Committee on Cancer; ER, estrogen receptor; PR, progesterone receptor; Her-2, human epidermal growth factor 2.

dietary habits and their disease spectrum also has its own salient features, such as high incidence of esophageal (11) and nasopharynx cancers (12). BC is the leading malignancy in females in China and increasingly threatens health with the same trend in southern China. In the present study, $78 \mathrm{BC}$ patients with T2DM in the Chaoshan area were compared to the randomly selected $300 \mathrm{BC}$ patients without T2DM. To the best of our knowledge, this is the first study to investigate the association between diabetes and $\mathrm{BC}$ in Chaoshannese.

A previous study has shown that obese patients had a 3-fold higher diabetes risk compared to non-obese patients (13). Approximately $50 \%$ of obese patients also suffer from diabetes, suggesting that obesity is a risk factor for the occurrence of T2DM. In the present study, $\leq 60 \%$ of BC patients with T2DM were at stage III. Reportedly, BMI is associated with $\mathrm{BC}$ recurrence and mortality by increasing the mortality risk of $\mathrm{BC}$ by 1.35 -fold for a BMI increment of $0.5 \sim 2.0 \mathrm{~kg} / \mathrm{m}^{2}$ and by 1.64 -fold at increments of $2.0 \mathrm{~kg} / \mathrm{m}^{2}$. In the present study, the proportion of overweight or obese $\mathrm{BC}$ patients with $\mathrm{T} 2 \mathrm{DM}$ is $\leq 50 \%$, similar to that reported in western countries (14).
As previously reported, there are no associations of diabetes with tumor size or lymph-node metastasis in $\mathrm{BC}$ patients $(15,16)$. However, BC patients with T2DM had more lymph nodes involvement and were at a more advanced TNM stage in the present study. These results demonstrate that T2DM may predict a worse prognosis in BC patients.

The direct biological influence of diabetes on $\mathrm{BC}$ is unknown. Li et al (16) reported that BC patients with T2DM had significantly lower DFS and OS than those without T2DM. Similarly, in the present study, the T2DM BC group had a significantly lower DFS and OS compared to the non-T2DM group. However, following adjustment for BMI, TNM stage and stratification of age, the DFS and OS did not significantly differ between BC patients with and without T2DM. Obesity, old age, concomitant diseases and treatment method are all relevant factors that confound the influence of diabetes to $\mathrm{BC}$.

The present study shows that in ER/PR-positive patients, the T2DM group has a higher recurrence compared to the non-T2DM group, although there is no difference in cancer-related mortalities. These results are consistent with a 
Table III. Cox proportional regression analysis on the overall survival rate of patients with invasive breast carcinoma stratified by diabetes status in the subgroups.

\begin{tabular}{|c|c|c|c|c|c|}
\hline Variables & T2DM group & Non-T2DM group & $\mathrm{HR}$ & $95 \% \mathrm{CI}$ & P-value \\
\hline \multicolumn{6}{|c|}{ Menopause status } \\
\hline Pre- & $5 / 25$ & $22 / 174$ & 1.967 & $0.719-5.380$ & 0.188 \\
\hline Post- & $14 / 53$ & $19 / 126$ & 1.474 & $0.709-3.061$ & 0.299 \\
\hline \multicolumn{6}{|c|}{$\mathrm{BMI}, \mathrm{kg} / \mathrm{m}^{2}$} \\
\hline$<25$ & $11 / 42$ & $33 / 230$ & 1.844 & $0.912-3.731$ & 0.089 \\
\hline$\geq 25$ & $8 / 36$ & $8 / 70$ & 1.410 & $0.507-3.200$ & 0.510 \\
\hline \multicolumn{6}{|l|}{ pT } \\
\hline $\mathrm{T}_{1-2}$ & $9 / 45$ & $22 / 208$ & 1.487 & $0.663-3.332$ & 0.335 \\
\hline $\mathrm{T}_{3-4}$ & $10 / 33$ & $19 / 92$ & 2.038 & $0.828-5.017$ & 0.121 \\
\hline \multicolumn{6}{|c|}{ Axillary lymph nodes } \\
\hline Negative & $2 / 25$ & $11 / 135$ & 0.824 & $0.167-4.067$ & 0.812 \\
\hline Positive & $17 / 53$ & $30 / 165$ & 1.875 & $0.995-3.532$ & 0.052 \\
\hline \multicolumn{6}{|c|}{ AJCC stage } \\
\hline I-II & $5 / 31$ & $14 / 180$ & 2.896 & $0.720-11.644$ & 0.134 \\
\hline III & $14 / 47$ & $27 / 120$ & 1.568 & $0.781-3.149$ & 0.206 \\
\hline \multicolumn{6}{|l|}{ Grade } \\
\hline 1 & $4 / 21$ & $9 / 100$ & 2.223 & $0.667-7.406$ & 0.193 \\
\hline $2-3$ & $15 / 57$ & $32 / 200$ & 1.472 & $0.767-2.824$ & 0.245 \\
\hline \multicolumn{6}{|c|}{ ER/PR status } \\
\hline Negative & $6 / 29$ & $18 / 94$ & 1.159 & $0.434-3.096$ & 0.768 \\
\hline Positive & $13 / 49$ & $23 / 206$ & 2.255 & $1.066-4.770$ & 0.033 \\
\hline \multicolumn{6}{|c|}{ Her-2 status } \\
\hline Negative & $12 / 49$ & $22 / 188$ & 2.033 & $0.961-4.300$ & 0.064 \\
\hline Positive & $7 / 29$ & 19/112 & 1.219 & $0.453-3.282$ & 0.695 \\
\hline Overall & $19 / 78$ & $41 / 300$ & 1.688 & $0.940-3.032$ & 0.079 \\
\hline
\end{tabular}

T2DM, type 2 diabetes mellitus; HR, hazard ratio; CI, confidence interval; BMI, body mass index; AJCC, American Joint Committee on Cancer; ER, estrogen receptor; PR, progesterone receptor; Her-2, human epidermal growth factor 2.

previous study by Schrauder et al (17). However, in the study by Schrauder et al, the ER-negative patients with T2DM also exhibited a higher distant metastasis risk than those without T2DM. ER/PR expression is an important predictor for therapeutic response and also a prognostic factor in $\mathrm{BC}$ patients. The present study demonstrates that T2DM is an independent factor of poor prognosis for ER/PR-positive BC patients. The poor prognosis in the ER/PR-positive subgroup of BC patients with T2DM may be attributed to limitation of choices for chemotherapeutic regimes due to diabetic complications (such as cardiovascular disease) and insensitivity to endocrine therapy due to increased free estrogen levels caused by downregulation of sex hormone-binding globulin in diabetic patients (18). At present, there are no standard treatment guidelines for ER-positive BC patients with T2DM. The currently used therapeutic dose of estrogen antagonist or aromatase inhibitors may be too low to compete with estrogen or to inhibit the transformation from androgen to estrogen. Therefore, for BC patients with T2DM, although ER-positivity indicates an improved prognosis, physicians should also focus more attention when choosing the optimized treatment strategy.
Although the underlying mechanism for the reason behind why T2DM is associated with a poorer prognosis remains undefined, the present findings provide evidence for administering more intensified adjuvant treatment for T2DM BC patients. There are certain limitations in the study: The number of cases studied was small and the follow-up period was short; post-operative treatment bias exists for patients with T2DM; and diabetes-targeted treatment influences BC prognosis.

In conclusion, $\mathrm{BC}$ patients with T2DM were significantly older, more likely to be overweight and had more lymph node involvement and more advanced TNM stage. The present study demonstrated that T2DM was associated with poor prognosis in ER/PR-positive BC patients.

\section{Acknowledgements}

The present study was partly supported by the funds from the Major State Basic Research Development Program of China (grant no. 2011CB707705), the Natural Science Foundation Committee (grant no. 31271068) and the Guangdong 
Provincial Key Laboratory on Breast Cancer Diagnosis and Treatment Research.

\section{References}

1. Danaei G, Finucane MM, Lu Y, et al: National, regional, and global trends in fasting plasma glucose and diabetes prevalence since 1980: systematic analysis of health examination surveys and epidemiological studies with 370 country-years and 2.7 million participants. Lancet 378: 31-40, 2011.

2. Giovannucci E, Harlan DM, Archer MC, et al: Diabetes and cancer: a consensus report. Diabetes Care 33: 1674-1685, 2010.

3. Bonovas S, Filioussi K and Tsantes A: Diabetes mellitus and risk of prostate cancer: a meta-analysis. Diabetologia 47: 1071-1078, 2004.

4. Ferlay J, Shin HR, Bray F, Forman D, Mathers C and Parkin DM: Estimates of worldwide burden of cancer in 2008: GLOBOCAN 2008. Int J Cancer 127: 2893-2917, 2010.

5. Larsson SC, Mantzoros CS and Wolk A: Diabetes mellitus and risk of breast cancer: a meta-analysis. Int J Cancer 121: 856-862, 2007.

6. Michels KB, Solomon CG, Hu FB, et al: Type 2 diabetes and subsequent incidence of breast cancer in the Nurses' Health Study. Diabetes Care 26: 1752-1758, 2003.

7. Chen WW, Shao YY, Shau WY, et al: The impact of diabetes mellitus on prognosis of early breast cancer in Asia. Oncologist 17: 485-491, 2012.

8. Liao S, Li J, Wang L, et al: Type 2 diabetes mellitus and characteristics of breast cancer in China. Asian Pac J Cancer Prev 11: 933-937, 2010.

9. Srokowski TP, Fang S, Hortobagyi GN and Giordano SH: Impact of diabetes mellitus on complications and outcomes of adjuvant chemotherapy in older patients with breast cancer. J Clin Oncol 27: 2170-2176, 2009.
10. Peairs KS, Barone BB, Snyder CF, et al: Diabetes mellitus and breast cancer outcomes: a systematic review and meta-analysis. J Clin Oncol 29: 40-46, 2011.

11. Li K and Yu P: Food groups and risk of esophageal cancer in Chaoshan region of China: a high-risk area of esophageal cancer. Cancer Invest 21: 237-240, 2003.

12. Zong YS: Nasopharyngeal carcinoma (NPC) mortality rate in two districts of Guangdong Province and their relation to $\operatorname{IgA}$ antibody level against EB virus capsid antigen. Zhonghua Zhong Liu Za Zhi 14: 103-105, 1992 (In Chinese).

13. Alberti KG and Zimmet PZ: Definition, diagnosis and classification of diabetes mellitus and its complications. Part 1: diagnosis and classification of diabetes mellitus provisional report of a WHO consultation. Diabet Med 15: 539-553, 1998.

14. Kroenke $\mathrm{CH}$, Chen WY, Rosner B and Holmes MD: Weight, weight gain, and survival after breast cancer diagnosis. J Clin Oncol 23: 1370-1378, 2005.

15. Guastamacchia E, Resta F, Mangia A, et al: Breast cancer: biological characteristics in postmenopausal type 2 diabetic women. Identification of therapeutic targets. Curr Drug Targets Immune Endocr Metabol Disord 3: 205-209, 2003.

16. Li Z, Luo Y, Gong Y, Liu Y, Qiu W and Tu J: Clinical features and molecular phenotypes of breast cancer in patients with type-2 diabetes mellitus. Asian Pac J Cancer Prev 12: 2183-2188, 2011.

17. Schrauder MG, Fasching PA, Haberle L, et al: Diabetes and prognosis in a breast cancer cohort. J Cancer Res Clin Oncol 137: 975-983, 2011.

18. Richardson LC and Pollack LA: Therapy insight: Influence of type 2 diabetes on the development, treatment and outcomes of cancer. Nat Clin Pract Oncol 2: 48-53, 2005. 